\title{
STABILITY INDICATING DETERMINATION OF DARUNAVIR WITH HPLC IN BLOOD PLASMA SAMPLES
}

\author{
Manchuru Vanaja and J. Sreeramulu* \\ Department of Chemistry, Sri Krishnadevaraya University, Anantapur, AP, India. \\ *E-mail: profjssku@yahoo.co.in
}

\begin{abstract}
Darunavir is an HIV/ AIDS treatment drug product. Darunavir is used with other medicines line cobicistat or ritonavir. The present objective is to develop and validate the HPLC method for the determination of darunavir in the blood plasma sample. Mobile Phase composed for $\mathrm{pH} 8.10$ buffer $\left(\mathrm{K}_{2} \mathrm{HPO}_{4}\right)$, Methanol and acetonitrile in the ratio of 488:162:350 v/v/v. The diluent is a mixture of water and acetonitrile 40:60 v/v. X-Bridge C18 5 $\mu \mathrm{m} 4.6 \times 250 \mathrm{~mm}$ column, wavelength $262 \mathrm{~nm}$, injection volume $20 \mu \mathrm{L}$, flow rate $1.3 \mathrm{ml} / \mathrm{min}$ and column oven temperature $35^{\circ} \mathrm{C}$ were used. Method validation was performed and results were within the acceptable limits. Results confirmed that the developed method is stability indicating and can be applied to analyze the blood plasma samples.

Keywords: Darunavir, blood plasma samples, tablets dosage form, method validation, antiretro viral drug product.

(C) RASĀYAN. All rights reserved
\end{abstract}

\section{INTRODUCTION}

Darunavir is used as an antiretroviral medication to control HIV / AIDS. This drug is recommended to use with other antiretroviral medicines. Darunavir was innovated by Tibotec at University of Illinois at Chicago $^{1-2}$. It can be used to control after a needle stick injury. Darunavir is approved in the USA in the year of 2006 and it is listed in WHO essential medicines. Darunavir drug is available in Darunavir/ Cobicistat, Darunavir/ Ritonavir combination single pill ${ }^{3-4}$. Darunavir is well tolerated by patients however it has side effects like high blood sugar, diabetes, muscle pain, tenderness, abdominal pain, and constipation, vomiting and headache ${ }^{5-6}$. Darunavir is available in ethanolate form and in the market, it is available in tablets dosage form with $75 \mathrm{mg}, 150 \mathrm{mg}$ and $600 \mathrm{mg}$.

Present research work objective was to develop a simple, stability indicating HPLC method for the quantification of darunavir in blood plasma and urine samples. Two known synthetic impurities were considered for the HPLC method. Chemical structure of darunavir and its impurities were represented in Fig.-1.

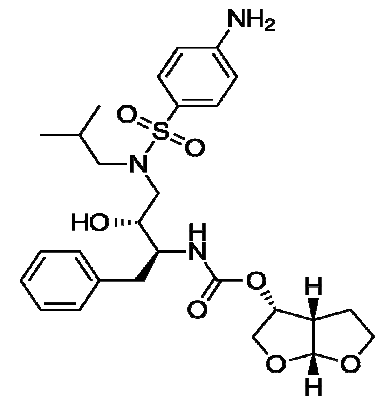

Darunavir

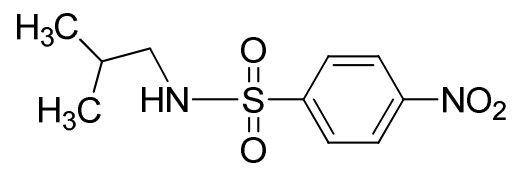

Impurity-1<smiles>CC(C)(C)OC(=O)N[C@@H](Cc1ccccc1)[C@H](O)CCl</smiles>

Impurity-2

Fig.-1: Chemical Structure of Darunavir and its Impurities

Darunavir: $\quad$ [(3aS,4R,6aR)-2,3,3a,4,5,6a-hexahydrofuro[2,3-b]furan-4-yl] $\quad$ N-[(2S,3R)-4-[(4aminophenyl)sulfonyl-(2-methylpropyl)amino]-3-hydroxy-1-phenylbutan-2-yl]carbamate

Rasayan J. Chem., 12(2), 839-848(2019)

http://dx.doi.org/10.31788/RJC.2019.1225118

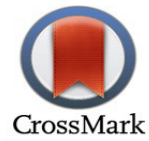


Impurity-1: N-isobutyl-4-nitrobenzenesulfonamide

Impurity-2: Tert-butyl ((2S,3S)-4-chloro-3-hydroxy-1-phenylbutan-2-yl)carbamate

Literature survey was performed and reported methods by HPLC/ MS are, Takahashi M (2007) ${ }^{7}$, Patel BN $(2012)^{8}$, Reddy BR $(2012)^{9}$, Babu GR $(2013)^{10}$, Satyanarayana L $(2011)^{11}$, Goldwirt L (2007) $)^{12}$. FTIR method by Kogawa AC $(2013)^{13}$. Main objective of this research work was to develop a simple, stability indicating HPLC method for the determination of darunavir in blood plasma and urine samples.

\section{Reagents}

\section{EXPERIMENTAL}

Di-potassium hydrogen phosphate anhydrous AR grade, Ortho phosphoric Acid AR grade, Acetonitrile HPLC Gradient Grade, Methanol HPLC Gradient Grade, Water for HPLC or equivalent.

\section{Preparation of Dilute Orthophosphoric Acid}

Diluted $1 \mathrm{~mL}$ of Orthophosphoric acid to $10 \mathrm{~mL}$ with water and mixed well.

\section{Preparation of Buffer Solution (pH 3.0)}

Weighed and transferred accurately about $4.35 \mathrm{~g}$ Di-potassium hydrogen phosphate anhydrous into $1000 \mathrm{~mL}$ water, sonicated to dissolve and mixed well. $\mathrm{pH}$ adjusted to $3.0 \pm 0.05$ with dilute Orthophosphoric acid. Mixed well and filtered through a $0.45 \mu$ membrane filter.

\section{Preparation of Mobile Phase}

4.35 g Dipotassium hydrogen phosphate anhydrous into $1000 \mathrm{~mL}$ water, sonicate to dissolve and mix well. Adjust $\mathrm{pH}$ to $8.10 \pm 0.05$ with dilute Orthophosphoric acid. $\mathrm{pH} 8.10$ buffer, Methanol and acetonitrile in the ratio of 488:162:350 v/v/v respectively.

\section{Preparation of Diluent}

Prepare a mixture of Water and Acetonitrile in the ratio of 40:60 v/v respectively.

\section{Chromatographic Conditions}

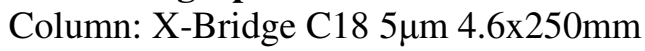

Wavelength: $262 \mathrm{~nm}$

Injection volume: $20 \mu \mathrm{L}$

Flow rate: $1.3 \mathrm{ml} / \mathrm{min}$

Column oven temperature: $35^{\circ} \mathrm{C}$

\section{Preparation of Diluted Standard Solution}

Weighed and transferred accurately 40mg of Darunavir standard into a $100 \mathrm{~mL}$ volumetric flask. 70 $\mathrm{mL}$ of diluent added and sonicated to dissolve and mix well. $10 \mathrm{~mL}$ of this solution pipetted into 100 $\mathrm{mL}$ volumetric flask and made up the volume to $50 \mathrm{~mL}$ with diluent and mixed well.

\section{Preparation of Sample Solution}

Sample powder equivalent to $40 \mathrm{mg}$ of darunavir weighed and transferred to $100 \mathrm{~mL}$ volumetric flask. $70 \mathrm{~mL}$ diluent added and sonicated for 30 minutes with intermittent shaking. Diluted up to the mark with diluent and mixed well. $10 \mathrm{ml}$ of solution pipetted and diluted to $100 \mathrm{ml}$ with diluent.

\section{Bioanalytical Sample}

The liquid-liquid extraction procedure was used to prepare plasma sample. Darunavir tablets 300mg were used; equivalent to $300 \mathrm{mg}$ of Darunavir test sample was spiked into $10 \mathrm{ml}$ plasma and stored at $2^{\circ} \mathrm{C}$ for 1 day. Stored spiked solutions were pull-out from the freezer and allowed reach room temperature. A $0.5 \mathrm{ml}$ aliquot was transferred to $10.0 \mathrm{~mL}$ polypropylene centrifuge tubes. $5.0 \mathrm{~mL}$ of ethyl acetate was used to get the two layers and extraction was completed. Centrifuge tubes were kept on vibramax unit for 15 min. Final solutions were centrifuged at $5000 \mathrm{rpm}$ for $5 \mathrm{~min}$ at $4^{\circ} \mathrm{C} .1 \mathrm{ml}$ of the supernatant solution was 
transferred into polypropylene tubes and evaporated at $40^{\circ} \mathrm{C}$. The dried residue was dissolved in $200 \mu \mathrm{L}$ of the mobile phase. Dilution was performed to reach $40 \mathrm{ppm}$ concentration. Samples, $20 \mu \mathrm{L}$ by volume, were then injected into the column and analyzed by HPLC on the same day to avoid any degradation. The column temperature oven was maintained at ambient temperature.

\section{Method Development}

\section{RESULTS AND DISCUSSION}

Literature survey and analytes nature were understood and initial method development were started with phosphate buffer and acetonitrile in the ratio of 60:40; inertsil C18 250mm, 4.6mm; 1.0 $\mathrm{ml} / \mathrm{min}$ flow rate; $20 \mu \mathrm{L}$ injection volume. Impurity-1 and 2 were eluted along with darunavir. Three components UV absorbance was scanned from 200 to $400 \mathrm{~nm}$ to select the maximum UV absorbance wavelength. Based on the UV absorbance spectrum for three analytes $267 \mathrm{~nm}$ was selected. Figure-2 represented the UV spectrum for darunavir and its impurities. Later we changed the mobile phase ratio with methanol and buffer $\mathrm{pH}$ also studied to get the high resolution between darunavir and impurities. Finally, pH8.1 buffer, acetonitrile and methanol mobile phase gave satisfactory results. Final method individual chromatograms were shown in Fig.-3 to 5.
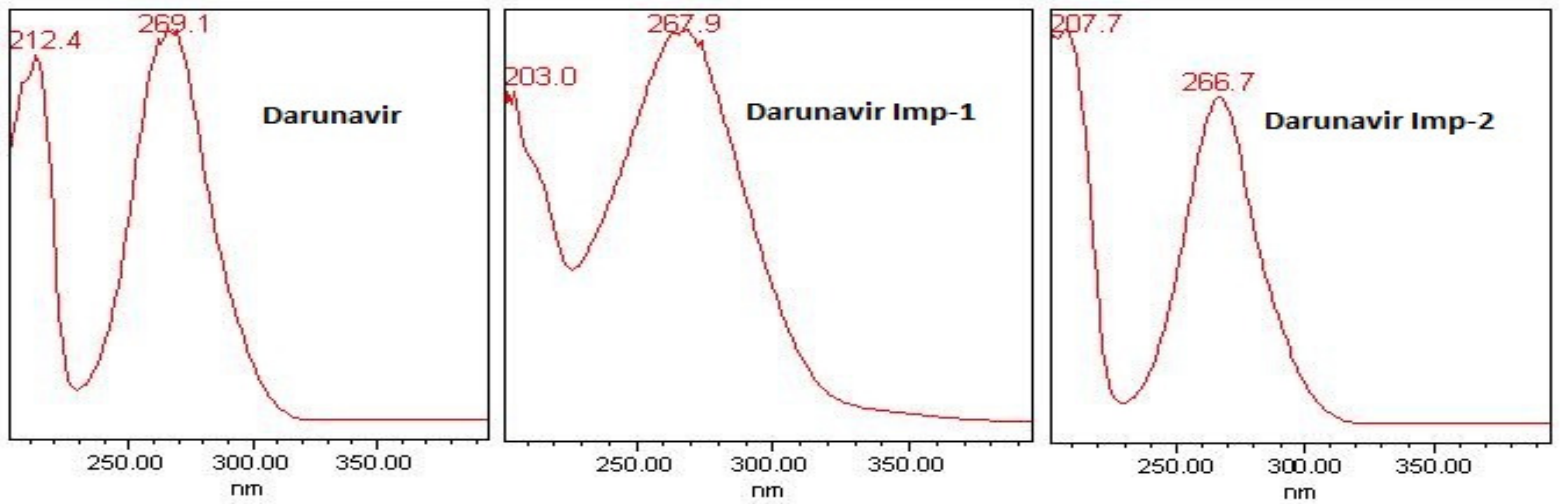

Fig.-2: UV Spectrum Darunavir and its Impurities 1 and 2

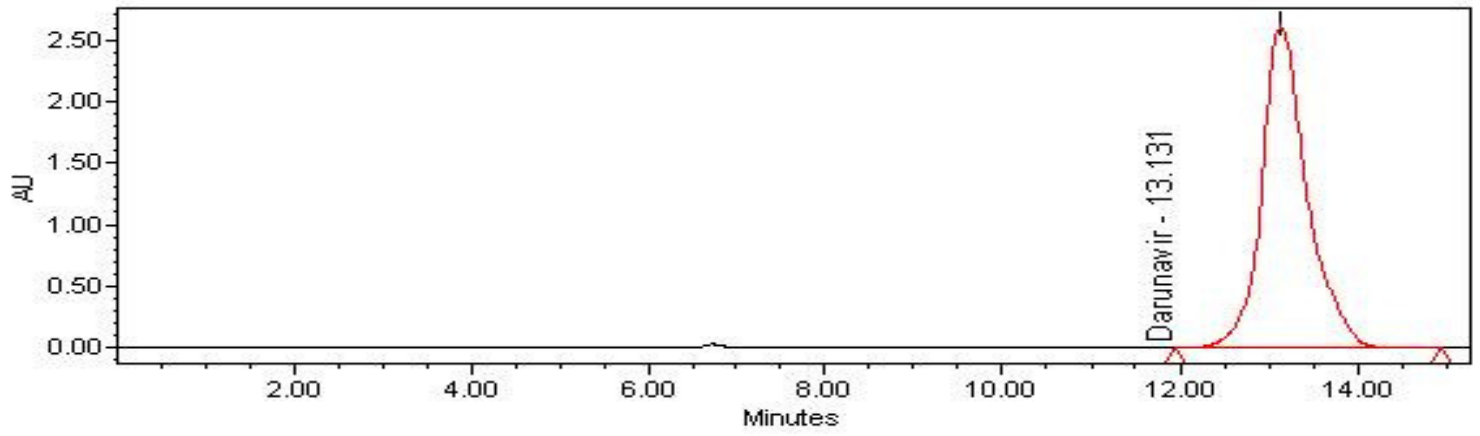

Fig.-3: Darunavir chromatogram

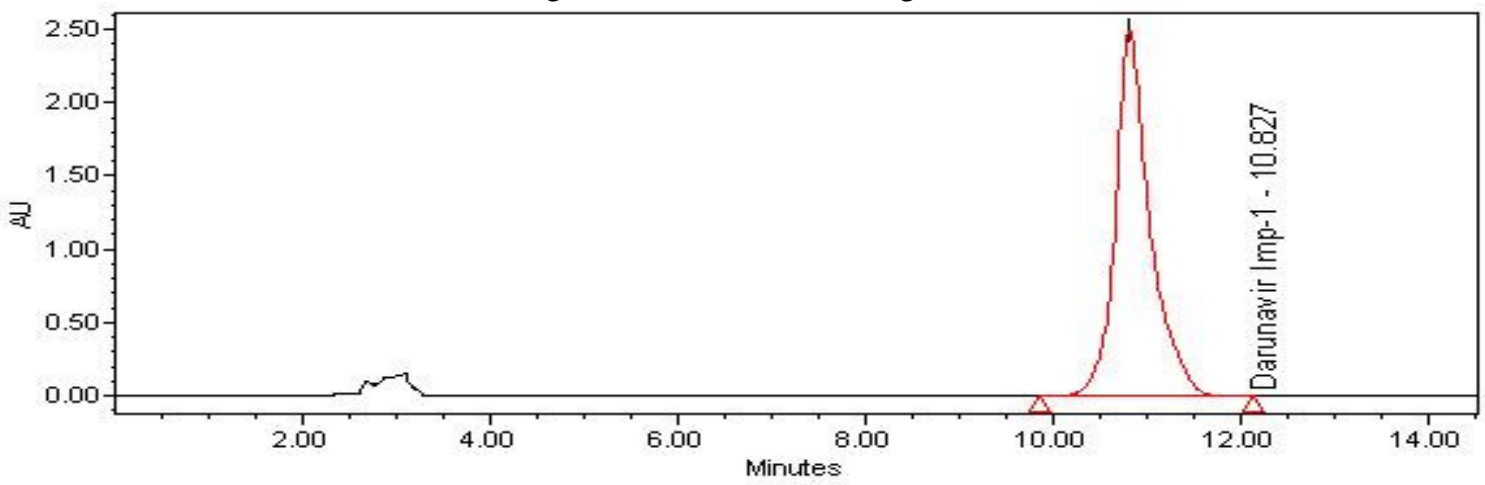

Fig.-4: Darunavir Impurity-1 Chromatogram 
RASĀYAN J. Chem.

Vol. 12 | No. 2 |839 - 848| April - June | 2019

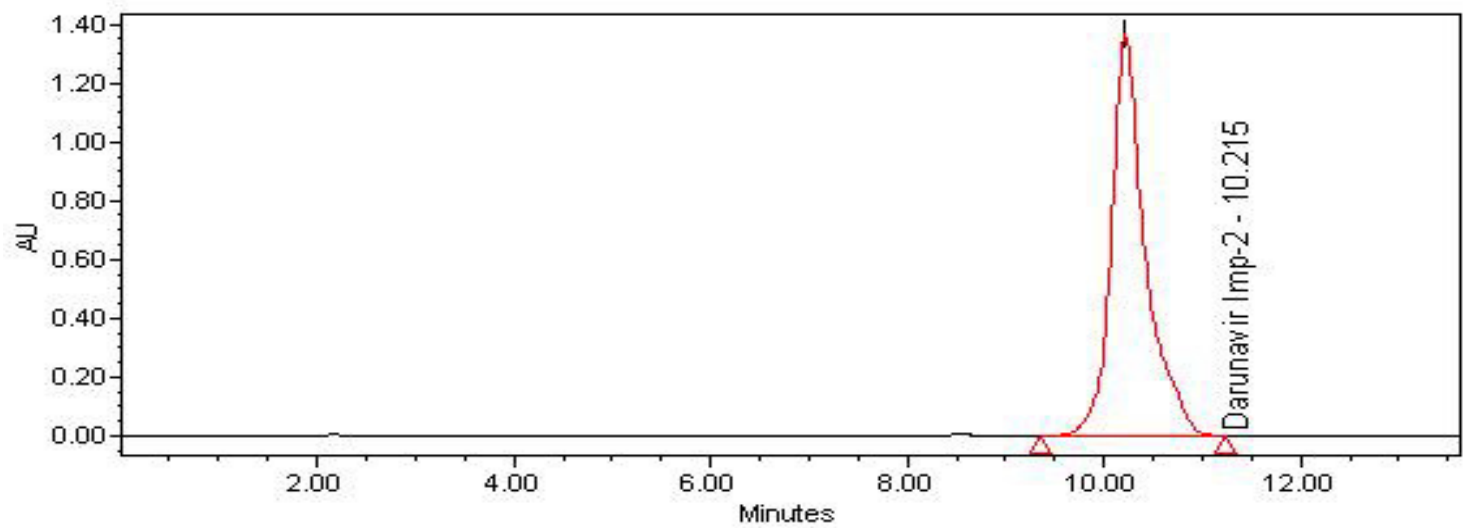

Fig.-5: Darunavir Impurity-2 Chromatogram

\section{System Suitability}

Final method was performed to confirm the method suitability and instrument suitability. FigureS-6, 7 and 8 represented the diluent, excipients solution and standard solution 5 injections. Table- 1 represents the system suitability results.

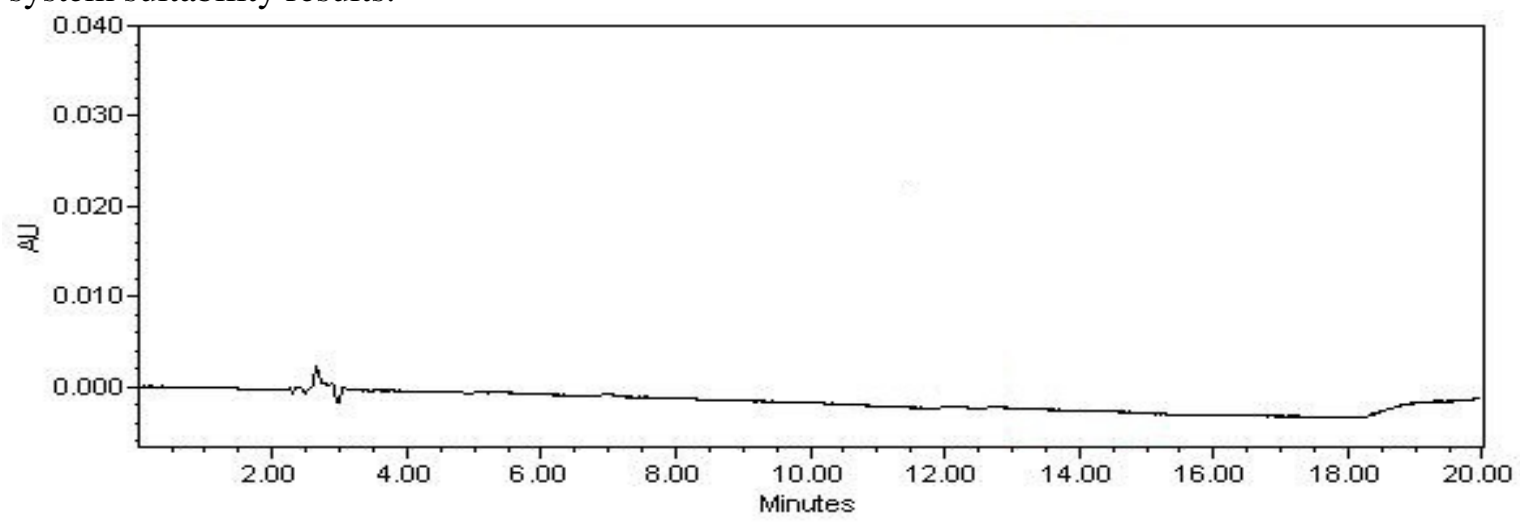

Fig.-6: Diluent Chromatogram

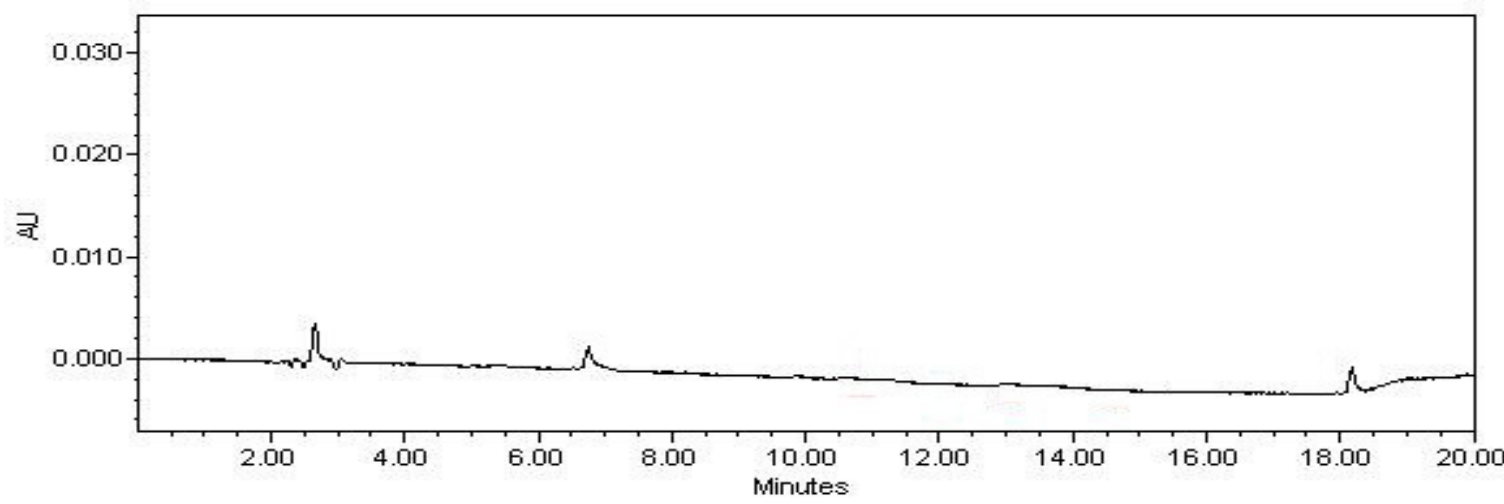

Fig.-7: Excipients Chromatogram

Table-1: System Suitability Results

\begin{tabular}{c|c|c|c}
\hline \multirow{2}{*}{ Injection } & \multicolumn{3}{|c}{ Darunavir } \\
\cline { 2 - 4 } & Retention time (min) & Area & Tailing factor \\
\hline 1. & 14.01 & 1545122 & 1.18 \\
\hline 2. & 14.08 & 1542314 & 1.15 \\
\hline 3. & 14.07 & 1531524 & 1.14 \\
\hline 4. & 14.06 & 1551241 & 1.16 \\
\hline 5. & 14.00 & 1543214 & 1.17 \\
\hline$\%$ RSD & 0.25 & 0.46 & NA \\
\hline
\end{tabular}


RASĀYAN J. Chem.

Vol. 12 | No. 2 |839 - 848| April - June | 2019
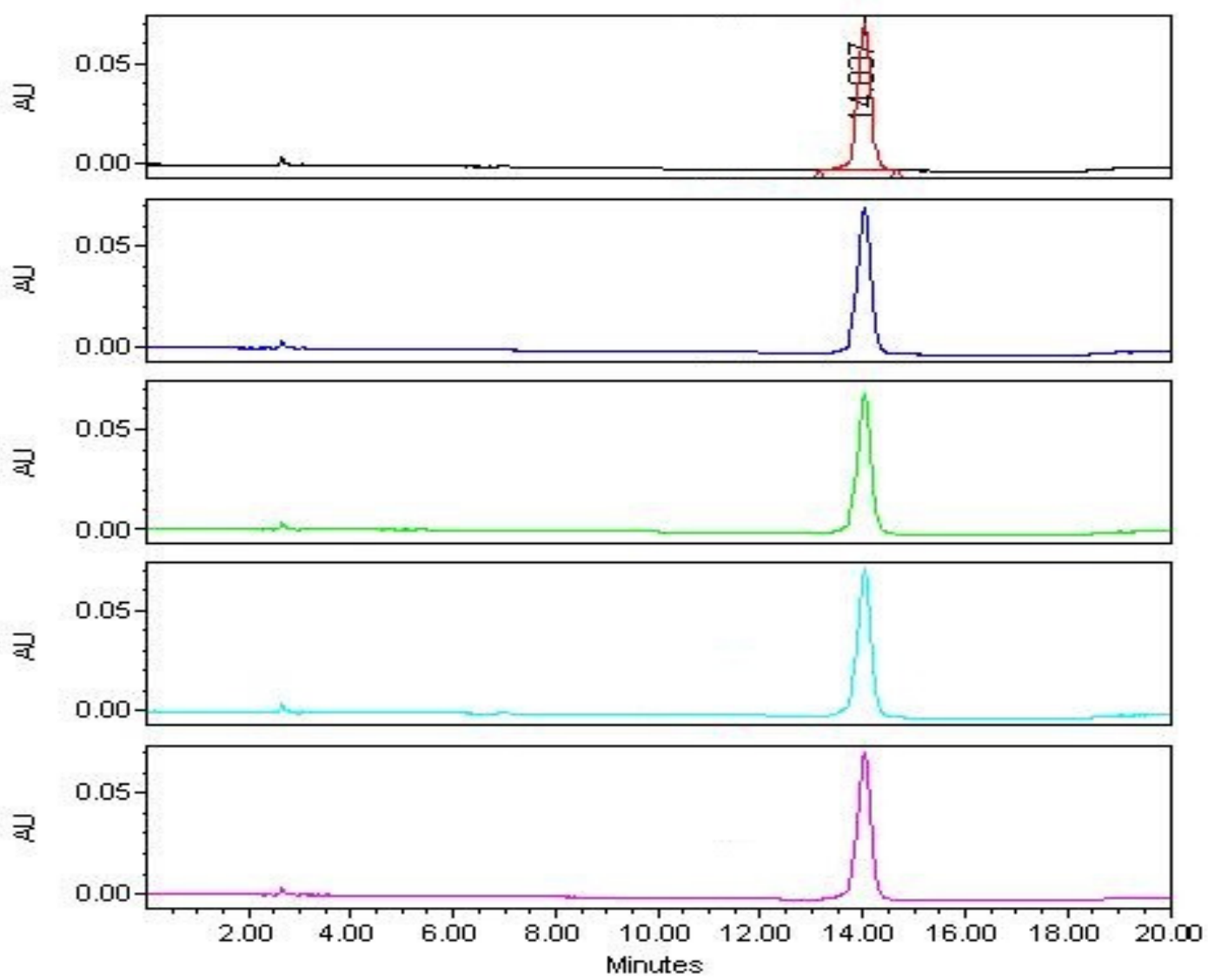

Fig.-8: Five Replicate Standard Solution Injections

Method validation was performed to confirm the method stability indicating property with specificity, precision, accuracy, ruggedness, robustness and linearity.

\section{Specificity}

Specificity was evaluated for the interference of blank, placebo and known impurities 1 and 2. Forced degradation studies were performed for acid, base, peroxide, thermal, water and UV light. Blank and placebo and known impurities-1 and 2 were well separated and no interference was observed at the retention time of darunavir. Figure- 9 t14 represented the force degradation chromatograms. Table- 2 represented the force degradation results and peak purity values.

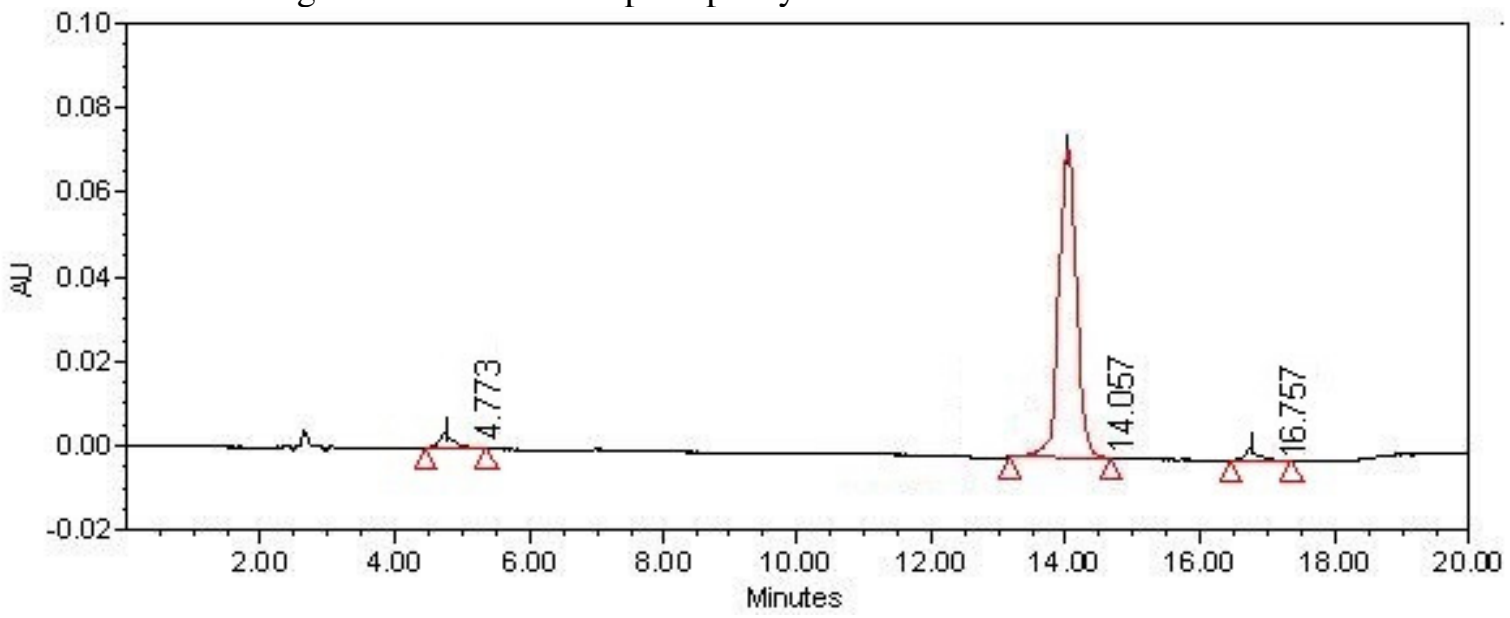

Fig.-9: Acid Degradation Sample Chromatogram 
RASĀYAN J. Chem.

Vol. 12 | No. 2 |839 - 848| April - June | 2019

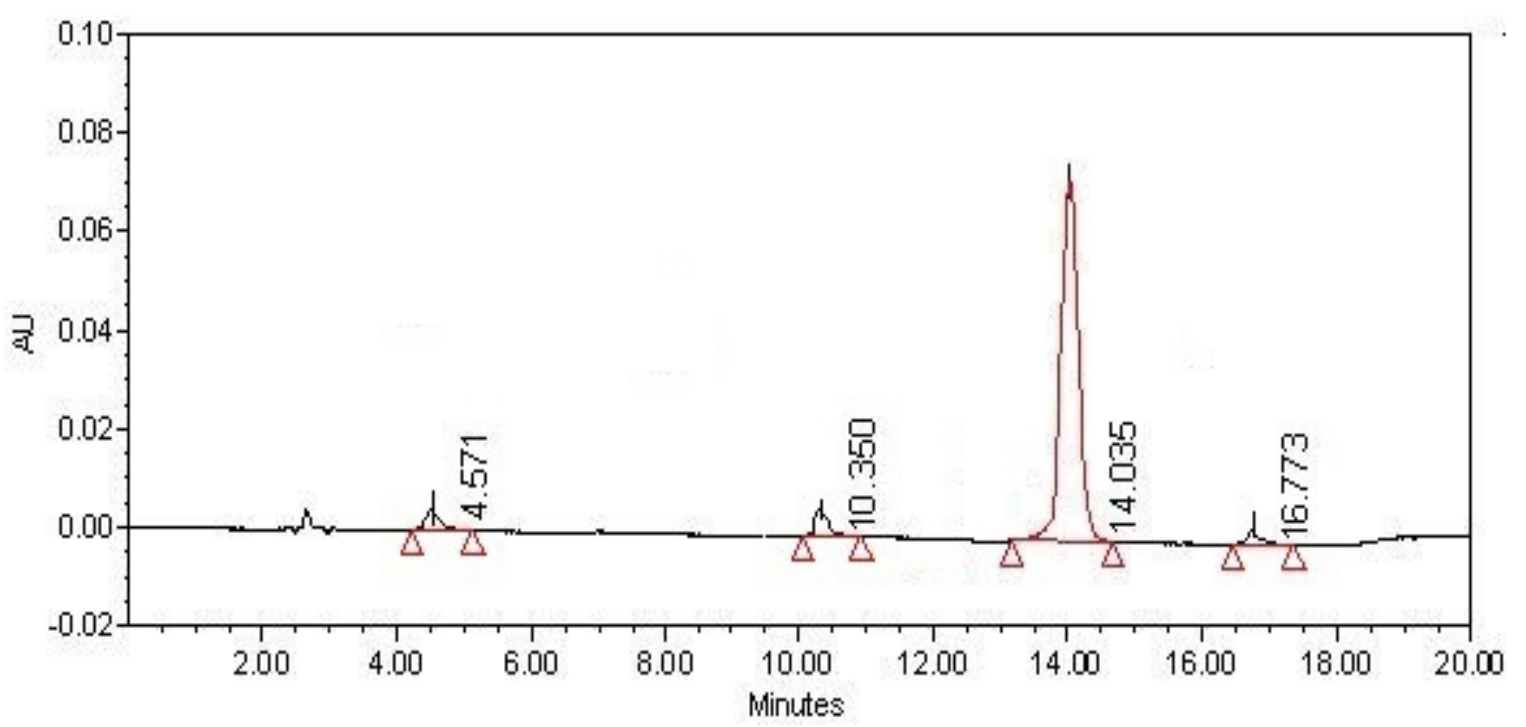

Fig.-10: Base Degradation Sample Chromatogram

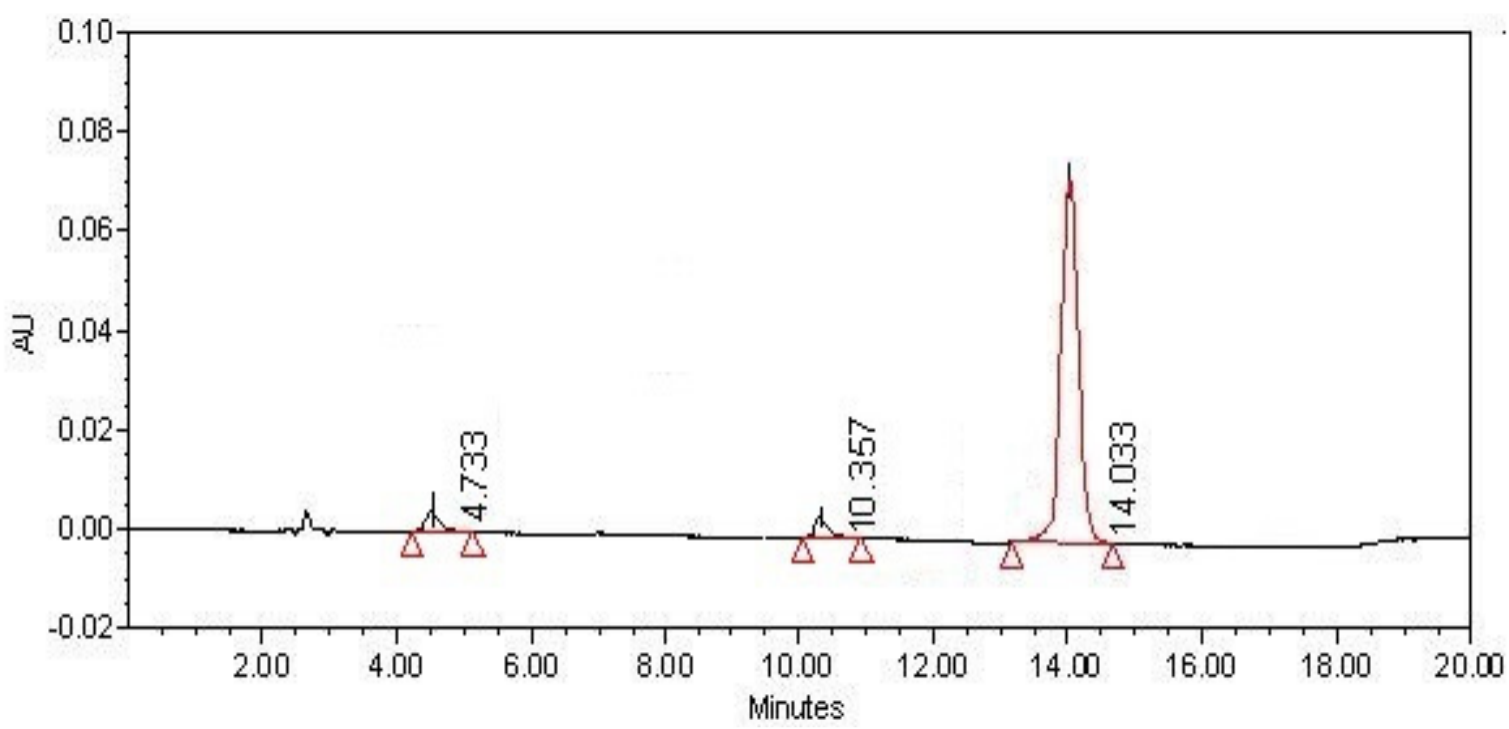

Fig.-11: Peroxide Degradation Sample Chromatogram

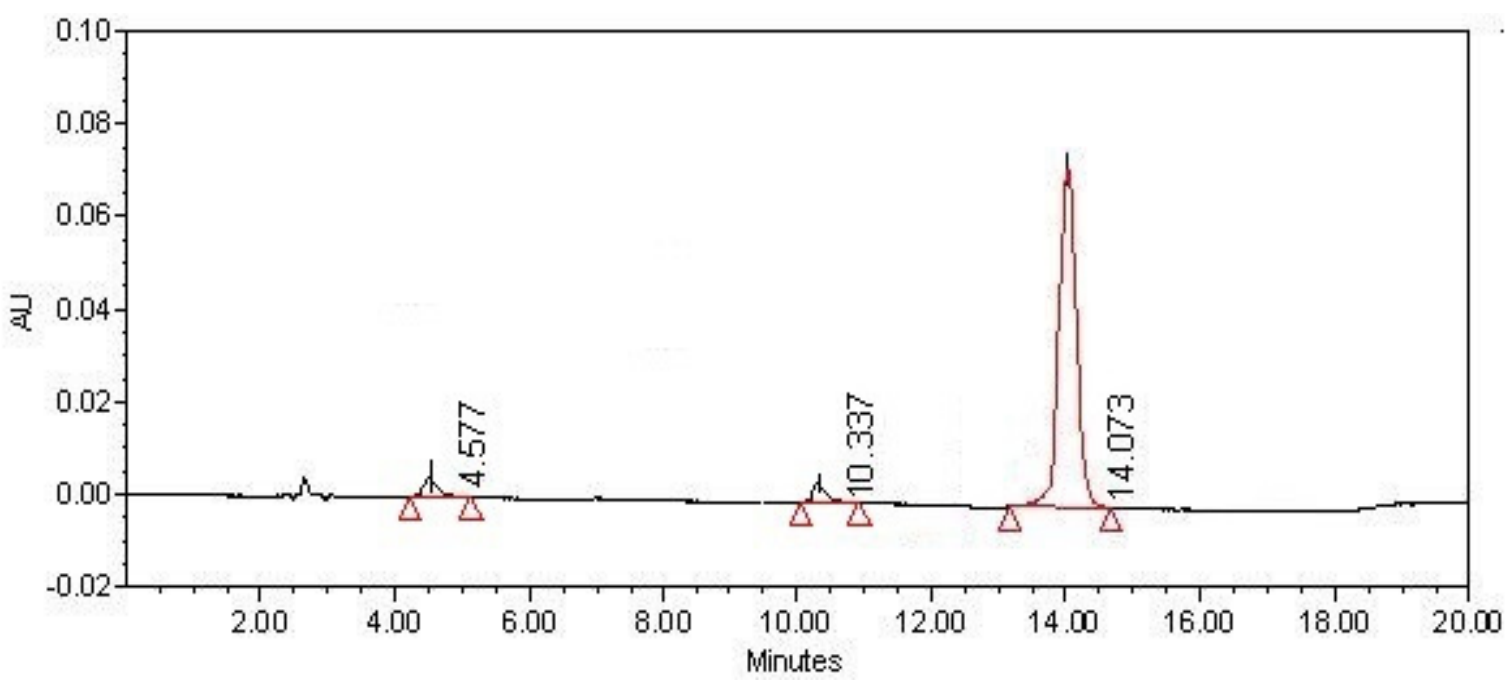

Fig.-12: Thermal Degradation Sample Chromatogram 
RASĀYAN J. Chem.

Vol. 12 | No. 2 |839 - 848| April - June | 2019

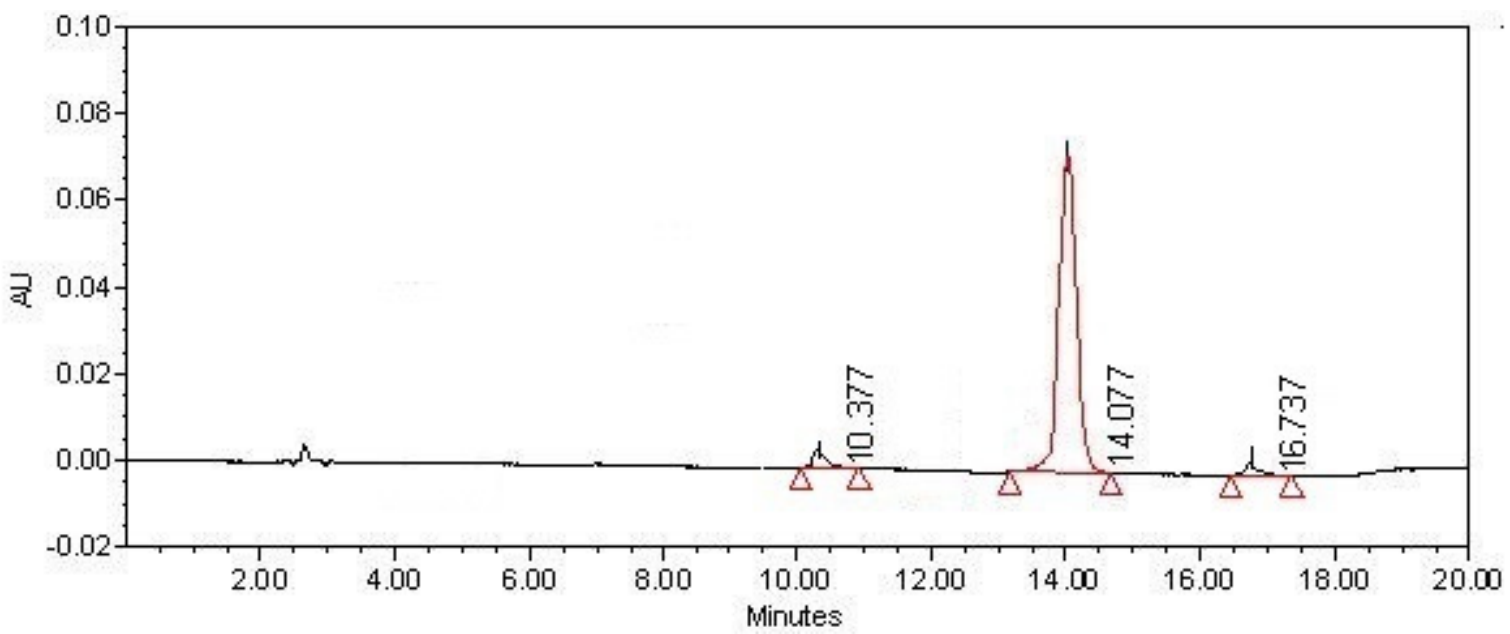

Fig.--13: UV/Visible Degradation Sample Chromatogram

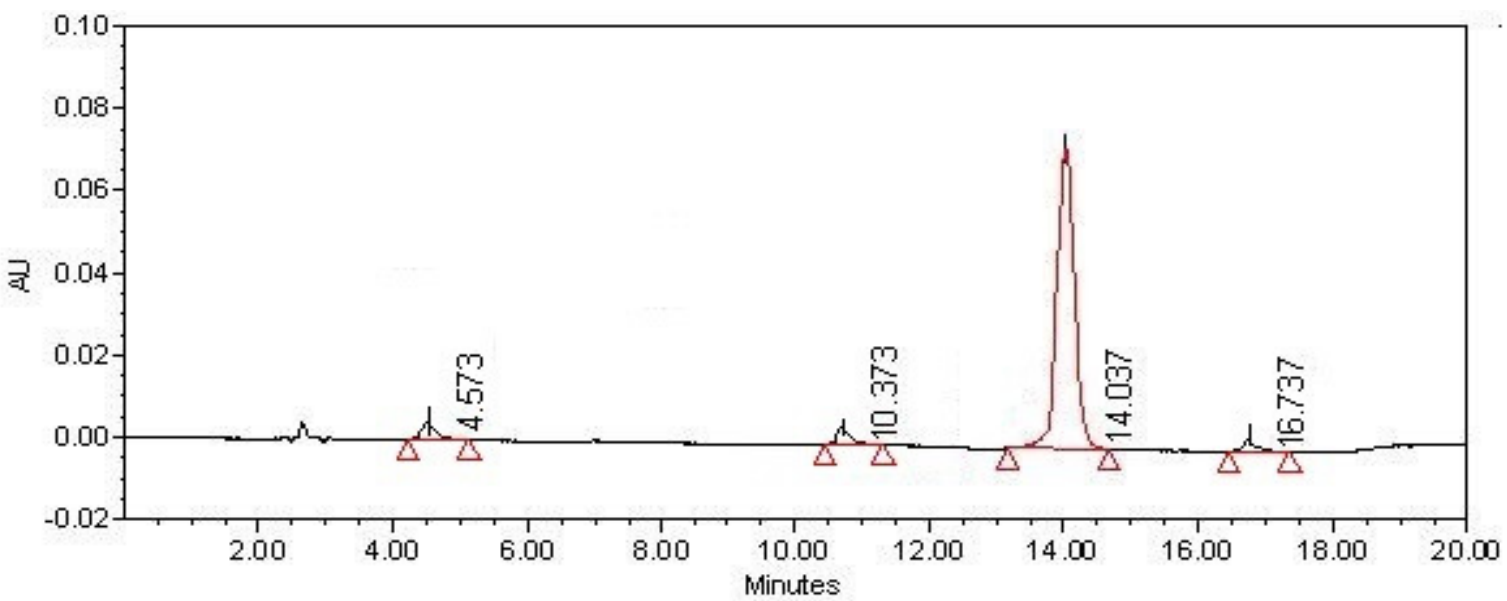

Fig.-14: Water Degradation Sample Chromatogram

Table-2: Stress Study Results

\begin{tabular}{c|c|c|c|c|c|c}
\hline S. No. & Degradation Condition & $\begin{array}{c}\text { Darunavir } \\
\text { Assay (\%) }\end{array}$ & $\begin{array}{c}\text { Purity } \\
\text { Angle }\end{array}$ & $\begin{array}{c}\text { Purity } \\
\text { Threshold }\end{array}$ & $\begin{array}{c}\text { Purity } \\
\text { Flag }\end{array}$ & Interference \\
\hline 1. & $\begin{array}{c}\text { Acid degradation } \\
\left(5 \mathrm{~N} \mathrm{HCl}, 1 \mathrm{hr}, 60^{\circ} \mathrm{C}\right)\end{array}$ & 97.04 & 0.204 & 0.391 & $\mathrm{NO}$ & $\mathrm{NO}$ \\
\hline 2. & $\begin{array}{c}\text { Base degradation } \\
\left(5 \mathrm{~N} \mathrm{NaOH}, 1 \mathrm{hr}, 60^{\circ} \mathrm{C}\right)\end{array}$ & 96.98 & 0.313 & 0.306 & $\mathrm{NO}$ & $\mathrm{NO}$ \\
\hline 3. & $\begin{array}{c}\text { Peroxide degradation } \\
\left(3 \% \mathrm{H}_{2} \mathrm{O}_{2}, 1 \mathrm{hr}, \mathrm{RT}\right)\end{array}$ & 97.89 & 0.241 & 0.440 & $\mathrm{NO}$ & $\mathrm{NO}$ \\
\hline 4. & $\begin{array}{c}\text { Water degradation } \\
\left(\text { Water, } 6 \mathrm{hr}, 60^{\circ} \mathrm{C}\right)\end{array}$ & 96.20 & 0.288 & 0.443 & $\mathrm{NO}$ & $\mathrm{NO}$ \\
\hline 5. & $\begin{array}{c}\text { Thermal degradation } \\
\left(24 \mathrm{hr}, 80^{\circ} \mathrm{C}\right)\end{array}$ & 97.25 & 0.276 & 0.430 & $\mathrm{NO}$ & $\mathrm{NO}$ \\
\hline 6. & $\begin{array}{c}\mathrm{UV} / \mathrm{visible} \mathrm{light} \\
(\mathrm{UV} \text { light } 200 \text { watt hr/sq. meter) } \\
(\text { Visible light } 1.2 \mathrm{million} \text { lux hrs })\end{array}$ & 96.80 & 0.228 & 0.420 & $\mathrm{NO}$ & $\mathrm{NO}$ \\
\hline
\end{tabular}

\section{Linearity}

Linearity was performed with five different concentration levels with freshly prepared solutions. Concentration levels $20 \mathrm{ppm}$ to $60 \mathrm{ppm}$ was performed. Linearity results were tabulated in below Table-3 and linearity overlay chromatograms were represented in Fig.-15. 
RASĀYAN J. Chem.

Vol. 12 | No. 2 |839 - 848| April - June | 2019

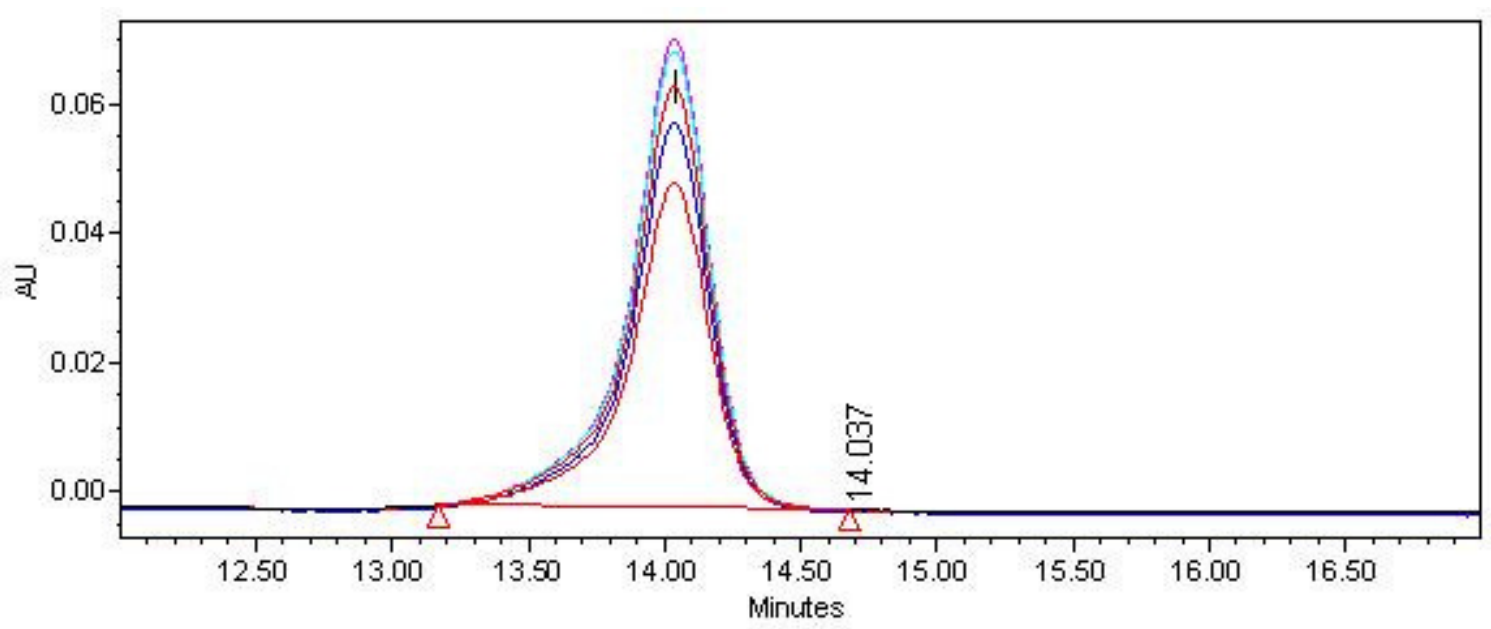

Fig.-15: Linearity Solutions Chromatograms Overlay View.

Table-3: Linearity Results

\begin{tabular}{l|l|l|l|l|l|l}
\hline Linearity Level & 1 & 2 & 3 & 4 & 5 & Coefficient of Correlation \\
\cline { 1 - 5 } Conc. $(\mu \mathrm{g} / \mathrm{ml})$ & 20 & 30 & 40 & 50 & 60 & 0.9993 \\
\cline { 1 - 5 } Area & 652580 & 113521 & 1625122 & 2215401 & 2681051 & \\
\hline
\end{tabular}

\section{Precision}

Method and system precision were performed with 6 replicate test solution preparations. Test solutions were prepared as per the test procedure mentioned in the material and methods section. \%RSD and \% assay were calculated and reported in table-4. \%RSD is within the acceptable limit i.e. 2.0 and \% assay values are between $98 \%$ and $102 \%$.

Table-4: Precision and Intermediate Precision Results

\begin{tabular}{c|c|c|c|c|c|c|c|c}
\hline \multicolumn{1}{c}{ Precision Results } \\
\hline S. No. & 1 & 2 & 3 & 4 & 5 & 6 & Average & \%RSD \\
\hline \% Assay & 99.63 & 99.67 & 99.89 & 99.84 & 98.99 & 99.19 & 99.55 & 0.33 \\
\hline \multicolumn{8}{c}{ Intermediate Precision } \\
\hline \% Assay & 99.87 & 99.28 & 99.59 & 99.67 & 99.82 & 99.68 & 99.69 & 0.22 \\
\hline
\end{tabular}

\section{Accuracy}

Accuracy was evaluated with 3 different concentration levels. A known concentration of sample was spiked to plasma sample and recovery studies were performed. Below Table-5 represented the accuracy results.

Table-5: Accuracy Results

\begin{tabular}{|c|c|c|c|c|c|c|c|c|c|c|c|c|c|}
\hline \multicolumn{14}{|c|}{ Darunavir Accuracy } \\
\hline$\mu g$ & Added & \multicolumn{3}{|c|}{20.12} & \multicolumn{6}{|c|}{40.23} & \multicolumn{3}{|c|}{60.31} \\
\hline$/ \mathrm{m}$ & $\begin{array}{c}\text { Recov } \\
.\end{array}$ & 20.16 & 20.61 & 20.31 & 40.20 & 40.15 & 40.19 & 40.31 & 40.29 & 40.31 & 60.19 & 60.21 & 60.19 \\
\hline \multicolumn{2}{|c|}{ \% Recovery } & 100.20 & 101.34 & 100.94 & 99.93 & 99.80 & 99.90 & 100.20 & 100.15 & 100.20 & 99.80 & 99.83 & 99.80 \\
\hline \multicolumn{2}{|c|}{$\begin{array}{c}\text { \% Recovery } \\
\text { Mean }\end{array}$} & \multicolumn{3}{|c|}{100.82} & \multicolumn{6}{|c|}{100.02} & \multicolumn{3}{|c|}{99.81} \\
\hline
\end{tabular}

\section{Ruggedness}

The ruggedness of the final method was checked with precision test samples. Precision sample-1 and 2 were stored at room temperature and refrigerator. Solutions stability was established for 3 days at room temperature and 5 days for refrigerator conditions. \% difference in the assay of darunavir and tailing factor, \%RSD was evaluated. Results were tabulated in Table-6. 
RASĀYAN J. Chem.

Vol. 12 | No. 2 |839 - 848| April - June | 2019

Table-6: Ruggedness Results

\begin{tabular}{|c|c|c|c|c|c|c|c|}
\hline \multirow{3}{*}{$\begin{array}{l}\text { Time in } \\
\text { Day }\end{array}$} & \multicolumn{4}{|c|}{ Bench Top Stability Test Solution } & \multirow[t]{3}{*}{ Tailing Factor } & \multirow[t]{3}{*}{$\% \mathrm{RSD}$} & \multirow{3}{*}{$\begin{array}{c}\text { Bench Top Stability } \\
\text { Standard Solution } \\
\text { Similarity factor }\end{array}$} \\
\hline & \multirow[t]{2}{*}{ Test-1 } & \multirow[t]{2}{*}{ Test-2 } & \multicolumn{2}{|c|}{ Difference } & & & \\
\hline & & & Test-1 & Test-2 & & & \\
\hline Initial & 99.63 & 99.67 & NA & NA & 1.10 & 0.5 & NA \\
\hline Day-1 & 99.29 & 99.35 & 0.34 & 0.32 & 1.16 & 1.8 & 1.10 \\
\hline \multirow[t]{2}{*}{ Day-3 } & 99.23 & 99.20 & 0.40 & 0.47 & 1.13 & 1.0 & 1.06 \\
\hline & \multicolumn{4}{|c|}{ Refrigerator Stability Test Solution } & & & $\begin{array}{c}\text { Refrigerator Stability } \\
\text { Standard Solution }\end{array}$ \\
\hline Initial & 99.63 & 99.67 & NA & NA & 1.13 & 0.5 & NA \\
\hline Day-3 & 99.59 & 99.45 & 0.04 & 0.22 & 1.15 & 1.0 & 1.05 \\
\hline Day-5 & 99.29 & 99.31 & 0.34 & 0.36 & 11 & 0.2 & 1.03 \\
\hline
\end{tabular}

\section{Robustness}

The ruggedness of the method was determined by verifying the chromatographic conditions such as flow rate, column temperature, mobile phase $\mathrm{pH}$, and acetonitrile and methanol ratio. Tailing factor and \% RSD for 5 standard solutions peak area were calculated and result acceptable (tailing factor not more than 2.0 and \%RSD is $2.0 \%$ ). Results were listed in Table-7 Filter verification was performed for PVDF and NYLON filters. Results were tabulated in Table-8.

Table-7: Results of Effect of Variations

\begin{tabular}{|c|c|c|c|c|c|c|}
\hline \multirow[b]{2}{*}{ Condition } & \multicolumn{3}{|c|}{ Flow Rate } & \multicolumn{3}{|c|}{ Column Temperature } \\
\hline & $1.1 \mathrm{ml} / \mathrm{min}$ & $1.3 \mathrm{ml} / \mathrm{min}$ & $1.5 \mathrm{ml} / \mathrm{min}$ & $30^{\circ} \mathrm{C}$ & $35^{\circ} \mathrm{C}$ & $40^{\circ} \mathrm{C}$ \\
\hline Tailing Factor & 1.1 & 1.1 & 1.1 & 1.1 & 1.1 & 1.1 \\
\hline \multirow[t]{3}{*}{$\%$ RSD for 5 Inj. } & 0.7 & 1.4 & 1.2 & 0.7 & 0.6 & 1.4 \\
\hline & \multicolumn{3}{|c|}{ Mobile Phase $\mathrm{pH}$} & \multicolumn{3}{|c|}{ M.P. Acetonitrile Ratio } \\
\hline & pH 7.9 & pH 8.1 & pH 8.3 & $100 \%$ & $90 \%$ & $110 \%$ \\
\hline Tailing Factor & 1.1 & 1.1 & 1.1 & 1.1 & 1.1 & 1.1 \\
\hline \multirow[t]{2}{*}{$\%$ RSD for 5 Inj. } & 0.7 & 1.4 & 1.5 & 0.7 & 0.4 & 1.0 \\
\hline & \multicolumn{3}{|c|}{ M.P. Acetonitrile Ratio } & & & \\
\hline Tailing Factor & \multirow{2}{*}{$100 \%$} & 1.3 & \multirow{2}{*}{$90 \%$} & 1.6 & \multirow[t]{2}{*}{$110 \%$} & 1.3 \\
\hline$\%$ RSD for 5 Inj. & & 0.6 & & 0.8 & & 0.9 \\
\hline
\end{tabular}

Table-8: Filter Variability Results

\begin{tabular}{c|c|c|c|c|c|c|c|c|c|c}
\hline \multirow{4}{*}{} & \multicolumn{2}{|c|}{ Centrifuged } & \multicolumn{3}{c|}{ Nylon Filter } & \multicolumn{4}{c}{ PVDF Filter } \\
\cline { 2 - 10 } & \multicolumn{2}{|c|}{$\%$ Assay } & \multicolumn{2}{|c|}{$\%$ Assay } & \multicolumn{2}{c}{ \% Difference } & \multicolumn{3}{c}{ \% Assay } & \% Difference \\
\cline { 2 - 10 } Darunavir & Spl-1 & Spl-2 & Spl-1 & Spl-2 & Spl-1 & Spl-2 & Spl-1 & Spl-2 & Spl-1 & Spl-2 \\
\cline { 2 - 11 } & 0.381 & 0.380 & 0.377 & 0.377 & 0.004 & 0.003 & 0.383 & 0.384 & 0.002 & 0.004 \\
\hline
\end{tabular}

\section{CONCLUSION}

Darunavir estimation in blood plasma sample was performed using RP-HPLC stability indicating method. HPLC method was developed by using a simple mobile phase with $\mathrm{pH}$ 8.1. Mobile phase composed of water, 
acetonitrile and methanol (488:162:350 v/v/v). X-Bridge C18 5 $\mu \mathrm{m} 4.6 \mathrm{x} 250 \mathrm{~mm}$ column, $262 \mathrm{~nm}$ wavelength, $20 \mu \mathrm{L}$ injection volume, $1.3 \mathrm{ml} / \mathrm{min}$ flow rate and column heater $35^{\circ} \mathrm{C}$ were applied. Method validation was performed with precision, accuracy, ruggedness, linearity, specificity and robustness. Results were within the acceptable limits such as tailing factor not more than 2.0, \% RSD not more than $2.0 \%$, linearity correlation coefficient 0.999 and \% recovery $98 \%$ to $102 \%$. The optimized method can be used to determine darunavir in plasma extraction samples.

\section{REFERENCES}

1. B. Clotet, N. Bellos, J.M. Molina, D. Cooper, J.C. Goffard, A. Lazzarin, A. Wöhrmann, C. Katlama, T. Wilkin, R. Haubrich, C. Cohen, The Lancet, 369(9568), 1169(2007), DOI: 10.1016/S01406736(07)60497-8

2. R. Ortiz, E. DeJesus, H. Khanlou, E. Voronin, J. van Lunzen, J. Andrade-Villanueva, J. Fourie, S. De Meyer, M. De Pauw, E. Lefebvre, T. Vangeneugden, Aids, 22(12), 1389(2008), DOI: 10.1097/QAD.0b013e32830285fb.

3. J.V. Madruga, D. Berger, M. McMurchie, F. Suter, D. Banhegyi, K. Ruxrungtham, D. Norris, E. Lefebvre, M.P. de Béthune, F. Tomaka, M. De Pauw, The Lancet, 370(9581), 49(2008), DOI: 10.1016/S0140-6736(07)61049-6

4. Y. Yazdanpanah, C. Fagard, D. Descamps, A.M. Taburet, C. Colin, B. Roquebert, C. Katlama, G. Pialoux, C. Jacomet, C. Piketty, D. Bollens, Clinical Infectious Diseases, 49(9), 1441(2009), DOI: $10.1086 / 630210$

5. J.R. Arribas, A. Horban, J. Gerstoft, G. Fätkenheuer, M. Nelson, N. Clumeck, F. Pulido, A. Hill, Y. Van Delft, T. Stark, C. Moecklinghoff, Aids, 24(2), 223(2010), DOI: 10.1097/QAD.0b013e3283348944

6. A.K. Ghosh, Z.L. Dawson, H. Mitsuya. Bioorganic \& medicinal chemistry, 15(24), 7576(2007), DOI: $10.1016 /$ j.bmc.2007.09.010

7. M. Takahashi, Y. Kudaka, N. Okumura, A. Hirano, K. Banno, T. Kaneda. Biological and Pharmaceutical Bulletin, 30(10), 1947(2007).

8. B.N. Patel, B.N. Suhagia, C.N. Patel, International Journal of Pharmacy and Pharmaceutical Sciences, 4(3), 270(2012).

9. B.R. Reddy, G. Jyothi, B.S. Reddy, N.V. Raman, K.S. Reddy, C. Rambabu, Journal of chromatographic science, 51(5), 471(2012).

10. G.R. Babu, A.L. Rao, J.V. Rao. Int J Res Pharm Chem., 3, 438(2013).

11. L. Satyanarayana, S.V. Naidu, M.N. Rao, A. Kumar, K. Suresh, Asian J Res Pharm Sci. 1, 74 (2011).

12. L. Goldwirt, S. Chhun, E. Rey, O. Launay, J.P. Viard, G. Pons, V. Jullien, Journal of Chromatography B, 857(2), 327(2007), DOI:10.1016/j.jchromb.2007.07.024

13. A.C. Kogawa, H.R. Salgado, Physical Chemistry, 3(1), 1-6 (2013), DOI: 10.4172/21532435.1000463

[RJC-5118/2018] 